\title{
Improved Bounds for Wireless Localization
}

\section{Journal Article}

\section{Author(s):}

Christ, Tobias; Hoffmann, Michael; Okamoto, Yoshio; Uno, Takeaki

Publication date:

2010

Permanent link:

https://doi.org/10.3929/ethz-b-000028772

Rights / license:

In Copyright - Non-Commercial Use Permitted

Originally published in:

Algorithmica 57(3), https://doi.org/10.1007/s00453-009-9287-2 


\title{
Improved Bounds for Wireless Localization
}

\author{
Tobias Christ • Michael Hoffmann • \\ Yoshio Okamoto • Takeaki Uno
}

Received: 22 August 2008 / Accepted: 28 January 2009 / Published online: 14 February 2009

(C) Springer Science+Business Media, LLC 2009

\begin{abstract}
We consider a novel class of art gallery problems inspired by wireless localization that has recently been introduced by Eppstein, Goodrich, and Sitchinava. Given a simple polygon $P$, place and orient guards each of which broadcasts a unique key within a fixed angular range. In contrast to the classical art gallery setting, broadcasts are not blocked by the edges of $P$. At any point in the plane one must be able to tell whether or not one is located inside $P$ only by looking at the set of keys received. In other words, the interior of the polygon must be described by a monotone Boolean formula composed from the keys. We improve both upper and lower bounds for the general problem where guards may be placed anywhere by showing that the maximum number of guards to describe any simple polygon on $n$ vertices is between roughly $\frac{3}{5} n$ and $\frac{4}{5} n$. A guarding that uses at most $\frac{4}{5} n$ guards can be obtained in $O(n \log n)$ time. For the natural setting where guards may be placed aligned to one edge or two consecutive edges of $P$ only, we prove that $n-2$ guards are always sufficient and sometimes necessary.
\end{abstract}

Keywords Computational geometry · Art gallery problems

\section{Introduction}

Art gallery problems are a classic topic in discrete and computational geometry, dating back to the question posed by Victor Klee in 1973: "How many guards are nec-

\footnotetext{
T. Christ $(\bowtie) \cdot$ M. Hoffmann

Institute for Theoretical Computer Science, ETH Zürich, Zürich, Switzerland

e-mail: tobias.christ@inf.ethz.ch

Y. Okamoto

Tokyo Institute of Technology, Tokyo, Japan

T. Uno

National Institute of Informatics, Tokyo, Japan
} 
essary, and how many are sufficient to patrol the paintings and works of art in an art gallery with $n$ walls?" Chvátal [2] was the first to show that $\lfloor n / 3\rfloor$ guards are always sufficient and sometimes necessary, while the beautiful proof of Fisk [6] made it into "the book" [1]. Nowadays there is a vast literature [13, 15, 17] about variations of this problem, ranging from optimization questions (minimizing the number of guards [11] or maximizing the guarded boundary [7]) over special types of guards (mobile guards [12] or vertex pi-guards [16]) to special types of galleries (orthogonal polygons [9] or curvilinear polygons [10]), to mention just a few classical and some more recent examples.

A completely different direction has recently been introduced by Eppstein, Goodrich, and Sitchinava [5]. They propose to modify the concept of visibility by not considering the edges of the polygon/gallery as blocking. This changes the problem quite drastically because it breaks up a certain locality where the shape of the polygon dictates the possible placement of guards.

The motivation for this model stems from communication in wireless networks where the signals are not blocked by walls, either. For illustration, suppose you run a café (modeled, say, as a simple polygon $P$ ) and you want to provide wireless Internet access to your customers. But you do not want the whole neighborhood to use your infrastructure. Instead, Internet access should be limited to those people who are located within the café. To achieve this, you can install a certain number of devices, let us call them guards, each of which broadcasts a unique (secret) key in an arbitrary but fixed angular range. The goal is to place guards and adjust their angles in such a way that everybody who is inside the café can prove this fact just by naming the keys received and nobody who is outside the café can provide such a proof. Formally this means that $P$ can be described by a monotone Boolean formula over the keys, that is, a formula using the operators AND and OR only, negation is not allowed.

It is convenient to model a guard as a subset of the plane, namely the area where the broadcast from this guard can be received. This area can be described as an intersection or union of at most two halfplanes. Using this notation, the polygon $P$ is to be described by a combination of the operations union and intersection over the guards. (See Fig. 1.)

Natural Guards Natural locations for guards are the vertices and edges of the polygon. A guard which is placed at a vertex of $P$ is called a vertex guard. A vertex guard is natural if it covers exactly the interior angle of its vertex. But natural vertex guards
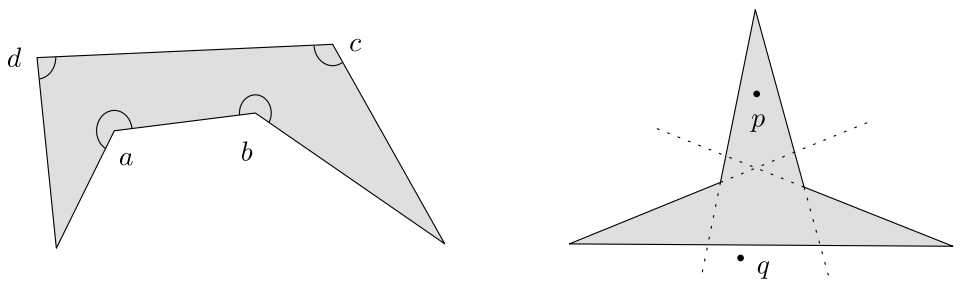

Fig. 1 A polygon described by $(a \cup b) \cap c \cap d$ and a polygon that cannot be guarded using natural vertex guards only 
alone do not always suffice [5], as the polygon shown in Fig. 1 to the right illustrates: No natural vertex guard can distinguish the point $p$ inside and the point $q$ outside the polygon. On the other hand, if $P$ is a convex polygon, putting a natural vertex guard on every second vertex is sufficient to describe $P$ as their intersection.

A guard placed anywhere on the line given by an edge of $P$ and broadcasting within an angle of $\pi$ to the inner side of the edge is called a natural edge guard. Of course, we can place a natural edge guard on one of the vertices of its incident edge. Hence a natural edge guard can always be realized as a (not necessarily natural) vertex guard. Dobkin, Guibas, Hershberger, and Snoeyink [4] showed that $n$ natural edge guards are sufficient for any simple polygon with $n$ edges.

Vertex Guards Eppstein et al. [5] proved that any simple polygon with $n$ edges can be guarded using at most $n-2$ (general, that is, not necessarily natural) vertex guards. More generally, they show that $n+2(h-1)$ vertex guards are sufficient for any simple polygon with $n$ edges and $h$ holes. This bound is not known to be tight. Damian, Flatland, O'Rourke, and Ramaswami [3] describe a family of simple polygons with $n$ edges which require at least $\lfloor 2 n / 3\rfloor-1$ vertex guards.

General Guards In the most general setting, we do not have any restriction on the placement and the angles of guards. So far the best upper bound known has been the same as for vertex guards, that is, $n-2$. On the other hand, if the polygon does not have collinear edges then at least $\lceil n / 2\rceil$ guards are always necessary [5]. The lower bound construction of Damian et al. [3] for vertex guards does not provide an improvement in the general case, where these polygons can be guarded using at most $\lceil n / 2\rceil+1$ guards. As O'Rourke wrote [14]: "The considerable gap between the $\lceil n / 2\rceil$ and $n-2$ bounds remains to be closed."

Results We provide a significant step in bringing the two bounds for general guards closer together by improving both on the upper and on the lower side. On one hand, there is an $O(n \log n)$-algorithm to construct a guarding using at most $\lfloor(4 n-2) / 5\rfloor$ guards for any given simple polygon with $n$ edges. The result easily generalizes to a finite number of polygons combined in some way by the operations intersection and/or union. In particular, any simple polygon with $h$ holes can be guarded using at most $\lfloor(4 n-2 h-2) / 5\rfloor$ guards. On the other hand, we describe a family of polygons which require at least $\lceil(3 n-4) / 5\rceil$ guards. Furthermore we obtain tight bounds for the case of natural guards. An extension of a result of Dobkin et al. [4] shows that $n-2$ natural (vertex or edge) guards are always sufficient. Somewhat surprisingly, it

Table 1 Number of guards needed for a simple polygon on $n$ vertices

\begin{tabular}{|c|c|c|c|c|c|c|c|c|}
\hline & \multicolumn{4}{|l|}{ Natural } & \multicolumn{4}{|l|}{ General } \\
\hline & vertex guards & & guards & & vertex guards & & guards & \\
\hline Upper bound & does not exist & {$[5]$} & $n-2$ & $\mathrm{a}$ & $n-2$ & {$[5]$} & $\lfloor(4 n-2) / 5\rfloor$ & a \\
\hline Lower bound & does not exist & {$[5]$} & $n-2$ & $\mathrm{a}$ & $\lfloor 2 n / 3\rfloor-1$ & [3] & $\lceil(3 n-4) / 5\rceil$ & a \\
\hline
\end{tabular}

${ }^{\mathrm{a}}$ Indicates the results of this paper 
turns out that this bound is tight. The same construction as for general guards yields a family of polygons which require $n-2$ natural (vertex or edge) guards.

The different problems and results are summarized in Table 1.

\section{Notation and Basic Properties}

We are given a simple polygon $P \subset \mathbb{R}^{2}$. A guard $g$ is a closed subset of the plane, whose boundary $\partial g$ is described by a vertex $v$ and two rays emanating from $v$ (see Fig. 2). The ray that has the interior of the guard to its right is called the left ray, the other one is called the right ray. The angle of a guard is the interior angle formed by its bounding rays. For a guard with angle $\pi$, the vertex is not unique.

A guard $g$ covers an edge $e$ of $P$ completely, if $e \subseteq \partial g$ and their orientations match, that is, the inner side of $e$ is on the inner side of $g$. We say $e$ is covered partly by $g$, if their orientations match and $e \cap \partial g$ is a proper sub-segment of $e$ that is not just a single point. We call a guard a $k$-guard, if it covers exactly $k$ edges completely. As $P$ is simple, a guard can cover at most one edge partly. If a guard covers an edge partly and $k$ edges completely, we call it a $k^{\prime}$-guard. Assuming there are no collinear edges, a guard can cover at most two edges; then a natural vertex guard is a 2-guard and a natural edge guard is a 1-guard.

The Wireless Localization Problem A guarding $\mathcal{G}(P)$ for $P$ is a formula composed of a set of guards and the operators union and intersection that defines $P$. The wireless localization problem is to find a guarding for a given simple polygon with as few guards as possible. The same problem is sometimes referred to as guard placement for point-in-polygon proofs or the sculpture garden problem [5]. The following statements are reformulations of results in [5].

Fig. 2 A guard $g$ with vertex $v_{g}$, left ray $\ell_{g}$ and right ray $r_{g}$ and a guard $g^{\prime}$ with its vertex and rays
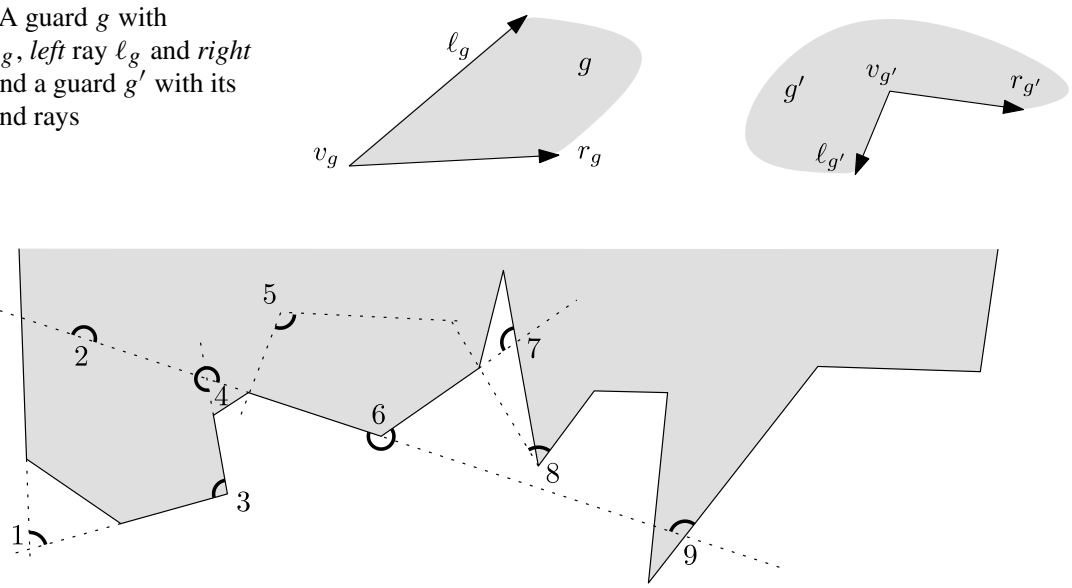

Fig. 3 Examples of guards: (1) a 2-guard, but not a vertex guard, (2) a 1-guard (and a natural edge guard), (3) a 2-guard (and a natural vertex guard), (4) a 2-guard, (5) a 0-guard, (6) a 0-guard (wrong orientation), (7) a 1-guard (and not a $1^{\prime}$-guard since the orientation is wrong), (8) a 1-guard (a non-natural vertex guard),

(9) a $1^{\prime}$-guard 
Observation 1 For any guarding $\mathcal{G}(P)$ and for any two points $p \in P$ and $q \notin P$ there is a guard $g \in \mathcal{G}(P)$ which distinguishes $p$ and $q$, that is, $p \in g$ and $q \notin g$.

Proof Suppose there is no such guard, that is, $p \in g$ implies $q \in g$ for all $g \in \mathcal{G}(P)$. Then the same holds for any union and intersection of guards from $\mathcal{G}(P)$. Therefore, no monotone formula built from the guards can describe $P$.

Lemma 2 In any guarding $\mathcal{G}(P)$, every edge of $P$ must be covered by at least one guard or it must be covered partly by at least two guards.

Proof Let $e$ be an edge of $P$. Suppose there is no guard that covers $e$ completely and at most one guard that covers $e$ partly. Then we can find a point $p \in e$ such that no guard ray passes through it. As $P$ is simple, there is a point $q$ close to $p$ and located outside $P$ such that every guard that contains $p$ contains $q$ as well, in contradiction to Observation 1.

Lemma 3 Any simple polygon with $n$ edges no two of which are collinear requires at least $\lceil n / 2\rceil$ guards.

Proof As there are no collinear edges, a guard can cover at most two edges (partly or completely). The bound follows from Lemma 2.

\section{Upper Bounds}

In this section we will derive upper bounds for the number of guards needed to cover any simple polygon. In fact, we obtain much more general results, which apply to any set that can be obtained from a finite collection of simple polygons by some combination of the operations intersection and union.

Following Dobkin et al. [4] we use the notion of a polygonal halfplane which is a topological halfplane bounded by a simple bi-infinite polygonal chain with edges $\left(e_{1}, \ldots, e_{n}\right)$, for $n \in \mathbb{N}$. For $n=1$, the only edge $e_{1}$ is a line and the polygonal halfplane is a halfplane. For $n=2, e_{1}$ and $e_{2}$ are rays which share a common source but are not collinear. Note that polygonal halfplanes with one or two edges are exactly the same as guards. For $n \geq 3, e_{1}$ and $e_{n}$ are rays, $e_{i}$ is a line segment, for $1<i<n$, and $e_{i}$ and $e_{j}$, for $1 \leq i<j \leq n$, do not intersect unless $j=i+1$ in which case they share an endpoint. For brevity we use the term chain in place of simple bi-infinite polygonal chain in the following.

For a polygonal halfplane $H$ define $\gamma(H)$ to be the minimum integer $k$ such that there exists a guarding $\mathcal{G}(H)$ for $H$ using $k$ guards. Similarly, for a natural number $n$, denote by $\gamma(n)$ the maximum number $\gamma(H)$ over all polygonal halfplanes $H$ that are bounded by a chain with $n$ edges. Obviously $\gamma(1)=\gamma(2)=1$. The results of Dobkin et al. [4] imply that $\gamma(n) \leq n$. Our main goal within this section is to improve this bound.

The following lemma makes the connection between guardings for polygonal halfplanes and simple polygons explicit. 
Lemma 4 Any simple polygon $P$ on $n \geq 4$ vertices can be expressed as an intersection of two polygonal halfplanes each of which consists of at least two edges.

Proof Let $p_{-}$and $p_{+}$be the vertices of $P$ with minimal and maximal $x$-coordinate, respectively. If they are not adjacent along $P$, split the circular sequence of edges of $P$ at both $p_{-}$and $p_{+}$to obtain two sequences of at least two segments each. Transform each sequence into a chain by linearly extending the first and the last segment beyond $p_{-}$or $p_{+}$(whichever of the two is incident) to obtain a ray. As $p_{-}$and $p_{+}$are opposite extremal vertices of $P$, the two chains intersect exactly at these two points (and nowhere else). Thus, the polygon $P$ can be expressed as an intersection of two polygonal halfplanes bounded by these chains.

Now consider the case that $p_{-}$and $p_{+}$are adjacent along $P$. Without loss of generality assume that $P$ lies above the edge from $p_{-}$to $p_{+}$. Rotate clockwise until another point $q$ has $x$-coordinate larger than $p_{+}$. If $q$ and $p_{-}$are not adjacent along $P$, then split $P$ at these points as described above. Otherwise the convex hull of $P$ is the triangle $q p_{-} p_{+}$. In particular, $q$ and $p_{+}$are opposite extremal vertices as well and they cannot be adjacent along $P$ because $P$ has more than three vertices. Therefore we can split at $q$ and $p_{+}$as described above.

The closure of the complement of a polygonal halfplane $H$, call it $\bar{H}$, is a polygonal halfplane as well. In particular, the closure of the complement of a guard $g$, denoted by $\bar{g}$, is a guard as well.

Observation 5 Any guarding for $H$ can be transformed into a guarding for $\bar{H}$ using the same number of guards.

Proof Use de Morgan's rules and invert all guards (keep their location but flip the angle to the complement with respect to $2 \pi$ ), see Fig. 4 .

Note that the resulting formula is indeed monotone because only guards complementary to the original ones appear (in SAT terminology: only negated literals); a formula is not monotone only if both a guard $g$ and its complementary guard $\bar{g}$ appear in it. In this way guarding the exterior of a simple polygon can be done in the same way as guarding its interior.

Our guarding scheme for chains is based on a recursive decomposition in which at each step the current chain is split into two or more subchains. At each split some
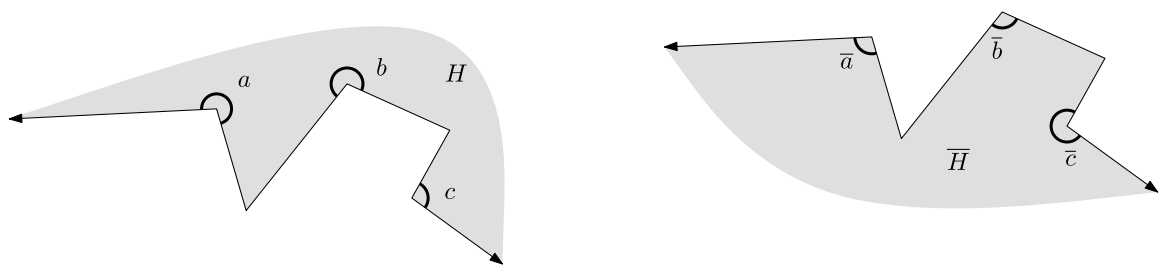

Fig. 4 A polygonal halfplane $H$ with a guarding $H=(a \cap b) \cup c$. Using de Morgan's rules we get a guarding of the complement $\bar{H}=\overline{(a \cap b) \cup c}=(\bar{a} \cup \bar{b}) \cap \bar{c}$ 
Fig. 5 A polygonal halfplane whose bounding chain $C$ has a degenerate convex hull $\mathrm{h}(C)$

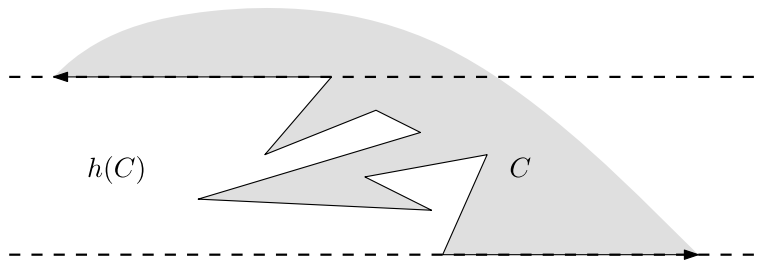

segments are extended to rays and we have to carefully control the way these rays interact with the remaining chain(s). This is particularly easy if the split vertex lies on the convex hull because then the ray resulting from the segment extension cannot intersect the remainder of the chain at all. However, we have to be careful what we mean by convex hull. As observed by Dobkin et al. [4], it is not the polygonal halfplane that matters but only its bounding chain. If the unbounded part of the halfplane (look at the plane from a point very high above) forms an angle greater than $\pi$, its convex hull is the entire plane. Only if the unbounded part of the halfplane forms a convex angle $(\leq \pi)$, its convex hull is bounded by a finite chain which starts and ends with a ray parallel to (possibly identical to) the rays of the chain bounding the halfplane. That said, instead of looking at the convex hull of a polygonal halfplane $H$ we work with the convex hull of its bounding chain $C$. The convex hull h $(C)$ of a chain $C=\left(e_{1}, \ldots, e_{n}\right)$, for $n \geq 2$, is either the convex hull of $H$ or the convex hull of $\bar{H}$, whichever of these two is not the whole plane, which solely depends on the direction of the two rays of $C$. The boundary of $\mathrm{h}(C)$ is denoted by $\partial \mathrm{h}(C)$. There is one degenerate case, when the two rays defining $C$ are parallel and all vertices are contained in the strip between them; in this case, $\mathrm{h}(C)$ is a strip bounded by the two parallel lines through the rays and thus $\partial \mathrm{h}(C)$ is disconnected (see Fig. 5).

\subsection{Natural Guards}

Theorem 6 Let $H$ be a polygonal halfplane bounded by a simple bi-infinite polygonal chain with $n \geq 2$ edges. Then $H$ can be guarded using at most $n-1$ natural guards.

Proof We proceed by induction on $n$. We follow the proof of Dobkin et al. [4] with the only difference in the base case: A chain with 2 edges can be guarded by one natural vertex guard. Now let $H$ be bounded by the chain $C$ with $n \geq 3$ edges.

Denote the sequence of edges along $C$ by $\left(e_{1}, \ldots, e_{n}\right)$ and let $v_{i}$, for $1 \leq i<n$, denote the vertex of $C$ incident to $e_{i}$ and $e_{i+1}$. The underlying (oriented) line of $e_{i}$, for $1 \leq i \leq n$, is denoted by $\ell_{i}$. For $2 \leq i \leq n-1$, let $e_{i}^{+}$be the ray obtained from $e_{i}$ by extending the segment linearly beyond $v_{i}$. Similarly $e_{i}^{-}$refers to the ray obtained from $e_{i}$ by extending the segment linearly beyond $v_{i-1}$. For convenience, let $e_{1}^{+}=\ell_{1}$ and $e_{n}^{-}=\ell_{n}$.

Let $v_{i}$ be a vertex on $\partial \mathrm{h}(C)$. Split $C$ at $v_{i}$ into two chains $C_{1}=\left(e_{1}, \ldots, e_{i}^{+}\right)$and $C_{2}=\left(e_{i+1}^{-}, \ldots, e_{n}\right)$. If $1<i<n-1$, then by induction there is a natural guarding $\mathcal{G}\left(C_{1}\right)$ using at most $i-1$ natural guards and a natural guarding $\mathcal{G}\left(C_{2}\right)$ with at most $n-i-2$ guards. So depending on $v_{i}$ being reflex or convex we obtain a natural 
guarding $\mathcal{G}\left(C_{1}\right) \cup \mathcal{G}\left(C_{2}\right)$ or $\mathcal{G}\left(C_{1}\right) \cap \mathcal{G}\left(C_{2}\right)$, respectively, using at most $n-2$ guards. In the special cases $i=1$ or $i=n-1$, that is, if $v_{i}$ is the first or last vertex of $C$ and one of the chains $C_{1}$ and $C_{2}$ is just a line, we still obtain a guarding using $n-1$ natural guards, because we can guard one chain with $n-2$ guards and the line with one natural edge guard.

As a consequence we obtain the following upper bound on the number of natural guards needed for a simple polygon. This bound turns out to be tight, as shown in Sect. 4. Observe that the statement is false for triangles which require two guards even without the restriction to natural guards.

Corollary 7 Any simple polygon $P$ with $n \geq 4$ edges can be guarded using at most $n-2$ natural (vertex or edge) guards.

Proof By Lemma $4 P$ can be described as an intersection of two polygonal halfplanes each of which consists of at least two edges. By Theorem 6 we can guard each of them by one guard less than it has edges.

Corollary 8 Let $P_{1}, \ldots, P_{m}$ be a collection of $m \geq 1$ simple polygons, $t$ of which are triangles, for $0 \leq t \leq m$. Let $R$ be a region that can be described as a formula composed of the operations intersection, union, and complement over the variables $\left\{P_{1}, \ldots, P_{m}\right\}$ in which each $P_{i}$ appears exactly once. Then $R$ can be guarded using at most $n-2 m+t$ natural (vertex or edge) guards, where $n$ is the total number of edges of the polygons $P_{i}$, for $1 \leq i \leq m$.

Also, one can easily treat polygons with holes and obtain a better bound as Eppstein et al. [5] give for general (not necessarily natural) guards. On the other hand, their result is slightly more general (triangles allowed) and stronger in the sense that the obtained formula is concise (a disjunction of conjunctions of constant size).

Corollary 9 Any simple polygon with $n \geq 4$ edges and $h$ non-triangular holes can be guarded using at most $n-2(h+1)$ natural (vertex or edge) guards.

\subsection{General Guards}

Theorem 10 Let $H$ be a polygonal halfplane bounded by a simple bi-infinite polygonal chain with $n \geq 2$ edges. Then a guarding for $H$ that uses at most $\lfloor(4 n-1) / 5\rfloor$ guards can be obtained in $O(n \log n)$ time.

Proof We first show the existence of a guarding with at most $\lfloor(4 n-1) / 5\rfloor$ guards by induction on $n$. The statement is easily checked for $2 \leq n \leq 3$. Let $C$ be any chain with $n \geq 4$ edges. We use the same notation as in the proof of Theorem 6 . Without loss of generality (cf. Observation 5) suppose that either the vertices of $C$ that lie on $\partial \mathrm{h}(C)$ are reflex, that is, $\mathrm{h}(C)$ is the convex hull of $\bar{H}$, or, in the degenerate case (see Fig. 5), that $v_{1}$ is reflex.

If there is any vertex $v_{i}$ on $\partial \mathrm{h}(C)$, for some $1<i<n-1$, then split $C$ into two chains $C_{1}=\left(e_{1}, \ldots, e_{i}^{+}\right)$and $C_{2}=\left(e_{i+1}^{-}, \ldots, e_{n}\right)$. We obtain a guarding for 
Fig. 6 The case where both $e_{1}$ and $e_{n}$ are part of $\partial \mathrm{h}(C)$. The small "brushes" mark the interior of the polygonal halfplane

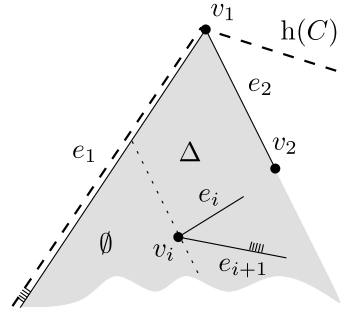

(a) Case 1 .

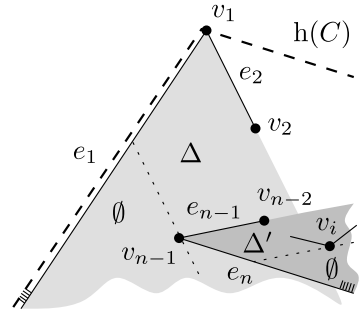

(b) Case 2 .

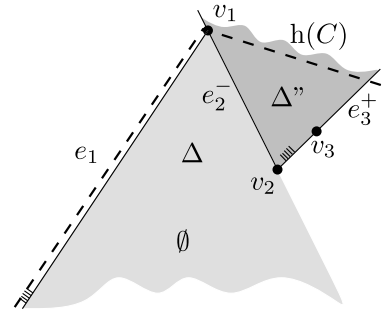

(c) Case 3 .

Fig. 7 The chain $C$ can interact with the shaded region $\Delta$ in three possible ways. The label $\emptyset$ marks an area which does not contain any vertex from $C$

$C$ as $\mathcal{G}\left(C_{1}\right) \cup \mathcal{G}\left(C_{2}\right)$ and thus $\gamma(C) \leq \gamma(i)+\gamma(n-i)$, for some $2 \leq i \leq n-2$. As both $i \geq 2$ and $n-i \geq 2$, we can bound by the inductive hypothesis $\gamma(C) \leq$ $\lfloor(4 i-1) / 5\rfloor+\lfloor(4 n-4 i-1) / 5\rfloor \leq\lfloor(4 i-1) / 5+(4 n-4 i-1) / 5\rfloor \leq\lfloor(4 n-1) / 5\rfloor$.

Else, if both $e_{1}$ and $e_{n}$ are part of $\partial \mathrm{h}(C)$ and $\ell_{1}$ intersects $\ell_{n}$, then we place a guard $g$ that covers both rays at the intersection of $\ell_{1}$ and $\ell_{n}$ to obtain a guarding $g \cup \mathcal{G}\left(e_{2}^{-}, \ldots, e_{n-1}^{+}\right)$for $C$ (see Fig. 6). Therefore, in this case $\gamma(C) \leq 1+\gamma(n-2)$. Observe that this is subsumed by the inequality from the first case with $i=2$.

Otherwise, either $\ell_{1}$ does not intersect $\ell_{n}$ and $v_{1}$ and $v_{n-1}$ are the only vertices of $\partial \mathrm{h}(C)$ (the degenerate case where $\partial \mathrm{h}(C)$ is disconnected, see Fig. 5) or without loss of generality (reflect $C$ if necessary) $v_{1}$ is the only vertex of $\partial \mathrm{h}(C)$. Let $\Delta$ denote the open (convex) wedge bounded by $e_{1}$ and $e_{2}^{+}$. We distinguish three cases.

Case 1 There is a vertex of $C$ in $\Delta$ and among these, a vertex furthest from $\ell_{2}$ is $v_{i}$, for some $3 \leq i \leq n-2$ (Fig. 7(a)). Split $C$ into three chains, $C_{1}=\left(\ell_{1}\right), C_{2}=$ $\left(e_{2}^{-}, \ldots, e_{i}^{+}\right)$, and $C_{3}=\left(e_{i+1}^{-}, \ldots, e_{n}\right)$. By the choice of $v_{i}$ there is no intersection between $C_{2}$ and $C_{3}$ other than at $v_{i}$. A guarding for $C$ can be obtained as $\mathcal{G}\left(C_{1}\right) \cup$ $\left(\mathcal{G}\left(C_{2}\right) \cap \mathcal{G}\left(C_{3}\right)\right)$. Therefore, in this case $\gamma(C) \leq 1+\gamma(j)+\gamma(n-j-1)$, for some $2 \leq j \leq n-3$. Since $j \geq 2$ and $n-j-1 \geq n-(n-3)-1=2$, we can apply the inductive hypothesis to bound $\gamma(C) \leq 1+\lfloor(4 j-1) / 5\rfloor+\lfloor(4 n-4 j-5) / 5\rfloor \leq$ $\lfloor(4 n-1) / 5\rfloor$.

Case 2 There is a vertex of $C$ in $\Delta$ and among these, the unique one furthest from $\ell_{2}$ is $v_{n-1}$ (Fig. 7(b)). We may suppose that $\ell_{1}$ intersects $\ell_{n}$; otherwise (the degenerate case where $\partial \mathrm{h}(C)$ is disconnected), exchange the roles of $v_{1}$ and $v_{n-1}$. We cannot 


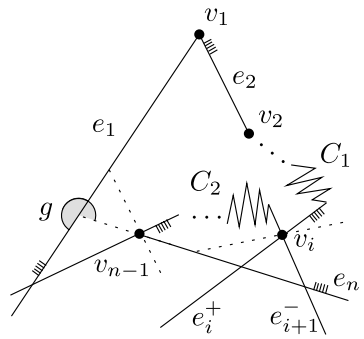

(a) Case 2.1

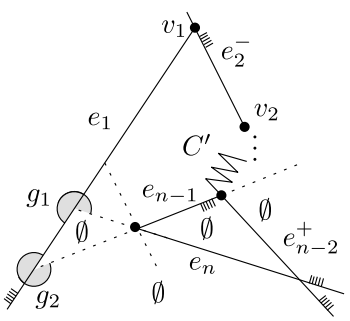

(b) Case 2.2.1

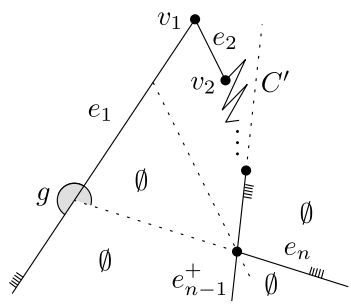

(c) Case 2.2 .2

Fig. 8 Either there is a vertex of $C$ in $\Delta^{\prime}$ or there is none. If there is none, we distinguish two subcases depending on whether $e_{n-1}^{+}$intersects $e_{1}$

end up in Case 2 both ways. Let $\Delta^{\prime}$ denote the open (convex) wedge bounded by $e_{n}$ and $e_{n-1}^{-}$.

If there is any vertex of $C$ in $\Delta^{\prime}$, let $v_{i}$ be such a vertex which is furthest from $\ell_{n-1}$ (see Fig. 8(a)). Split $C$ into two chains, $C_{1}=\left(e_{1}, \ldots, e_{i}^{+}\right)$and $C_{2}=\left(e_{i+1}^{-}, \ldots, e_{n-1}^{+}\right)$. Both $C_{1}$ and $C_{2}$ are simple, except that their first and their last ray may intersect (in that case split the resulting polygon into two chains). Put a guard $g$ at the intersection of $\ell_{n}$ with $e_{1}$ such that $g$ covers $e_{n}$ completely and $e_{1}$ partially. A guarding for $C$ can be obtained as $g \cap\left(\mathcal{G}\left(C_{1}\right) \cup \mathcal{G}\left(C_{2}\right)\right)$. Again this yields $\gamma(C) \leq 1+\gamma(i)+\gamma(n-i-1)$, for some $2 \leq i \leq n-3$, and thus $\gamma(C) \leq\lfloor(4 n-1) / 5\rfloor$ as above in Case 1 .

Otherwise there is no vertex of $C$ in $\Delta^{\prime}$. We distinguish two sub-cases. If $e_{n-1}^{+}$ intersects $e_{1}$ then put two guards (see Fig. 8(b)): a first guard $g_{1}$ at the intersection of $\ell_{n}$ with $e_{1}$ such that $g_{1}$ covers $e_{n}$ completely and $e_{1}$ partially, and a second guard $g_{2}$ at the intersection of $\ell_{n-1}$ with $e_{1}$ such that $g_{2}$ covers $e_{n-1}$ completely and $e_{1}$ partially. Together $g_{1}$ and $g_{2}$ cover $e_{1}$ and $g_{1} \cap\left(g_{2} \cup \mathcal{G}\left(C^{\prime}\right)\right)$ provides a guarding for $C$, with $C^{\prime}=\left(e_{2}^{-}, \ldots, e_{n-2}^{+}\right)$. In this case we obtain $\gamma(C) \leq 2+\gamma(n-3)$ and thus by the inductive hypothesis $\gamma(C) \leq 2+\lfloor(4 n-13) / 5\rfloor \leq\lfloor(4 n-1) / 5\rfloor$.

Finally, suppose that $e_{n-1}^{+}$does not intersect $e_{1}$ (see Fig. 8(c)). Then for the chain $C^{\prime}=\left(e_{1}, \ldots, e_{n-1}^{+}\right)$there is some vertex other than $v_{1}$ on the convex hull boundary $\mathrm{h}\left(C^{\prime}\right)$. Thus we can obtain a guarding for $C^{\prime}$ as described above for the case that there is more than one vertex on the convex hull. Put a guard $g$ at the intersection of $\ell_{n}$ with $e_{1}$ such that $g$ covers $e_{n}$ completely and $e_{1}$ partially. This yields a guarding $g \cap \mathcal{G}\left(C^{\prime}\right)$ for $C$ with $\gamma(C) \leq 1+\gamma\left(C^{\prime}\right) \leq 1+\gamma(i)+\gamma(n-i-1)$, for some $2 \leq i \leq n-3$. As in Case 1 we conclude that $\gamma(C) \leq\lfloor(4 n-1) / 5\rfloor$.

Case 3 There is no vertex of $C$ in $\Delta$ (Fig. 7(c)). Let $\Delta^{\prime \prime}$ denote the open (convex) wedge bounded by $e_{2}^{-}$and $e_{3}^{+}$. If $e_{3}^{-}$does not intersect $e_{1}$ then put a natural vertex guard $g$ at $v_{1}$ to obtain a guarding $g \cap \mathcal{G}\left(C^{\prime}\right)$ for $C$, where $C^{\prime}=\left(e_{3}^{-}, \ldots, e_{n}\right)$. This yields $\gamma(C) \leq 1+\gamma(n-2)$ and thus by the inductive hypothesis $\gamma(C) \leq$ $1+\lfloor(4 n-9) / 5\rfloor \leq\lfloor(4 n-1) / 5\rfloor$.

Now suppose that $e_{3}^{-}$intersects $e_{1}$. We distinguish two subcases. If there is no vertex of $C$ in $\Delta^{\prime \prime}$, then place two guards: a natural vertex guard $g_{1}$ at $v_{1}$ and a guard $g_{2}$ at the intersection of $e_{3}^{-}$with $e_{1}$ such that $g_{1}$ covers $e_{3}$ completely and $e_{1}$ partially (see Fig. 9(a)). A guarding for $C$ is provided by $g_{1} \cap\left(g_{2} \cup \mathcal{G}\left(C^{\prime}\right)\right)$, with 


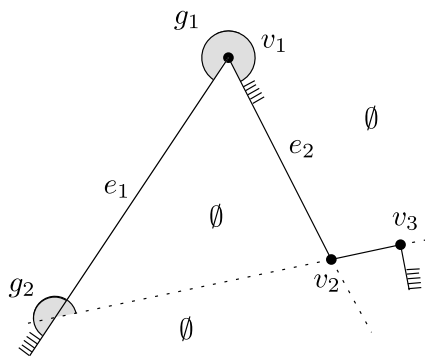

(a) Case 3.1

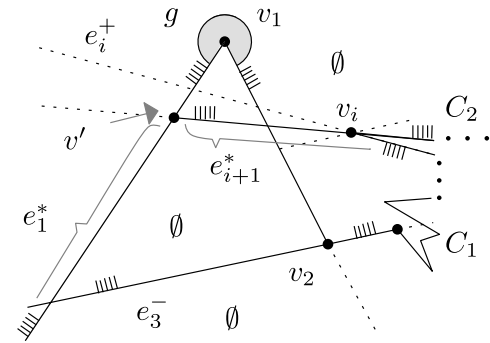

(b) Case 3.2

Fig. 9 The subcase where $\Delta^{\prime \prime}$ is empty and the subcase where it is not

$C^{\prime}=\left(e_{4}^{-}, \ldots, e_{n}\right)$. In this case we obtain $\gamma(C) \leq 2+\gamma(n-3)$ and thus in the same way as shown above $\gamma(C) \leq\lfloor(4 n-1) / 5\rfloor$.

Otherwise there is a vertex of $C$ in $\Delta^{\prime \prime}$. Let $v_{i}$, for some $4 \leq i \leq n-1$, be a vertex of $C$ in $\Delta^{\prime \prime}$ which is furthest from $\ell_{3}$. First suppose $e_{i+1}^{-}$does not intersect $e_{2}$. Then neither does $e_{i}^{+}$and hence we can split at $v_{i}$ in the same way as if $v_{i}$ would be on $\partial \mathrm{h}(C)$. If $i=n-1, e_{n}^{-}$must intersect $e_{2}$ (otherwise, $e_{n}$ would be on $\partial \mathrm{h}(C)$ ). Thus we have $i<n-1$ and both chains consist of at least two segments/rays.

Now suppose that $e_{i+1}^{-}$intersects $e_{2}$ and thus $e_{1}$, and denote the point of intersection between $e_{i+1}^{-}$and $e_{1}$ by $v^{\prime}$. Let $e_{1}^{*}$ be the ray originating from $v^{\prime}$ in direction $e_{1}$, and let $e_{i+1}^{*}$ denote the segment or ray (for $i=n-1$ ) originating from $v^{\prime}$ in direction $e_{i+1}^{-}$. Place a natural vertex guard $g$ at $v_{1}$. See Fig. 9(b). Regardless of whether or not $e_{i}^{+}$intersects $e_{2}$ and $e_{1}$, a guarding for $C$ is provided by $g \cap\left(\mathcal{G}\left(C_{1}\right) \cup \mathcal{G}\left(C_{2}\right)\right)$, with $C_{1}=\left(e_{3}^{-}, \ldots, e_{i}^{+}\right)$and $C_{2}=\left(e_{1}^{*}, e_{i+1}^{*} \ldots, e_{n}\right)$ (if $i=n-1$ then $\left.C_{2}=\left(e_{1}^{*}, e_{n}^{*}\right)\right)$. Observe that by the choice of $v_{i}$ both $C_{1}$ and $C_{2}$ are simple and $\gamma(C) \leq 1+\gamma(j)+\gamma(n-j-1)$, for some $2 \leq j \leq n-3$. As above, this yields $\gamma(C) \leq\lfloor(4 n-1) / 5\rfloor$.

We have shown that in every case $\gamma(C) \leq\lfloor(4 n-1) / 5\rfloor$ and as $C$ was arbitrary it follows that $\gamma(n) \leq\lfloor(4 n-1) / 5\rfloor$. (One might be tempted to believe that the same analysis yields a better upper bound of $\lfloor(2 n-1) / 3\rfloor$. But note that this bound does not hold for $n=3$, which is the reason why the proof would break down.)

The above analysis yields a recursive algorithm to construct a guarding using at most $\lfloor(4 n-1) / 5\rfloor$ guards. It remains to prove the claimed running time. Store the input chain $C$ as an array $\left(e_{1}, \ldots, e_{n}\right)$ of its edges. Each edge $e_{i}$ in turn is represented by its direction $d_{i}$ and its target vertex $v_{i}$ (the latter being undefined for $e_{n}$ ). A subchain $\left(e_{i}, \ldots, e_{j}\right)$ of $C$ is represented by its bounding indices $i$ and $j$.

Apart from constant time geometric primitives, such as testing whether two given rays intersect, the algorithm needs to find an extreme point among a contiguous subsequence $V_{i, j}:=\left(v_{i}, \ldots, v_{j}\right)$, for some $1 \leq i \leq j<n$, of vertices from $C$. Using a compact interval tree [8] on the vertices of $C$, we can find extreme points for any $V_{i, j}$, $1 \leq i \leq j<n$, in $O(\log n)$ time after $O(n \log n)$ preprocessing. No other ingredients are needed for the algorithm, any test whether a certain region is empty boils down to an extreme point query on a suitably chosen subsequence of vertices. For instance, to test whether the region $\Delta$ is empty of points in Case 1, it is enough to know the 
extreme point of $V_{3, n}$ in direction $e_{1}$. Therefore, any single step of the algorithm can be handled in $O(\log n)$ time. As in each step the current chain is split, the number of steps is linear and the overall runtime is $O(n \log n)$.

Corollary 11 For any simple polygon $P$ with $n$ edges a guarding using at most $\lfloor(4 n-2) / 5\rfloor$ guards can be obtained in $O(n \log n)$ time.

Proof Triangles can be guarded with two guards and for $n \geq 4$ the bound follows from Lemma 4 and Theorem 10.

Corollary 12 Let $P_{1}, \ldots, P_{m}$ be a collection of $m \geq 1$ simple polygons with $n$ edges in total, and let $R$ be a region that can be described as a formula composed of the operations intersection, union, and complement over the variables $\left\{P_{1}, \ldots, P_{m}\right\}$ in which each $P_{i}$ appears exactly once. Then $R$ can be guarded using at most $\lfloor(4 n-$ $2 m) / 5\rfloor$ guards.

Corollary 13 Let $P$ be any simple polygon with h holes such that $P$ is bounded by $n$ edges in total. Then $P$ can be guarded using at most $\lfloor(4 n-2 h-2) / 5\rfloor$ guards.

\section{Lower Bounds}

For any natural number $m$ we construct a polygon $P_{m}$ with $2 m$ edges which requires "many" guards. The polygon consists of spikes $S_{1}, S_{2}, \ldots, S_{m}$ arranged in such a way that the lines through both edges of a spike cut into every spike to the left (see Fig. 10).

Denote the apex of $S_{i}$ by $w_{i}$ and its left vertex by $v_{i}$. The edge from $v_{i}$ to $w_{i}$ is denoted by $e_{i}$, the edge from $w_{i}$ to $v_{i+1}$ by $f_{i}$. We can construct $P_{m}$ as follows: Consider the two hyperbolas $\left\{(x, y) \in \mathbb{R}^{2} \mid x \geq 1, y=\frac{1}{x}\right\}$ and $\left\{(x, y) \in \mathbb{R}^{2} \mid x \geq 1, y=-\frac{1}{x}\right\}$. Let $v_{1}:=(1,1)$ and $w_{1}:=(1,-1)$. Then choose $f_{1}$ tangential to the lower hyperbola. Let $v_{2}$ be the point where the tangent of the lower hyperbola intersects the upper hyperbola, that is, $v_{2}=\left(1+\sqrt{2}, \frac{1}{1+\sqrt{2}}\right)$. Choose $w_{2}$ to be the point where the tangent

Fig. 10 Example consisting of four spikes

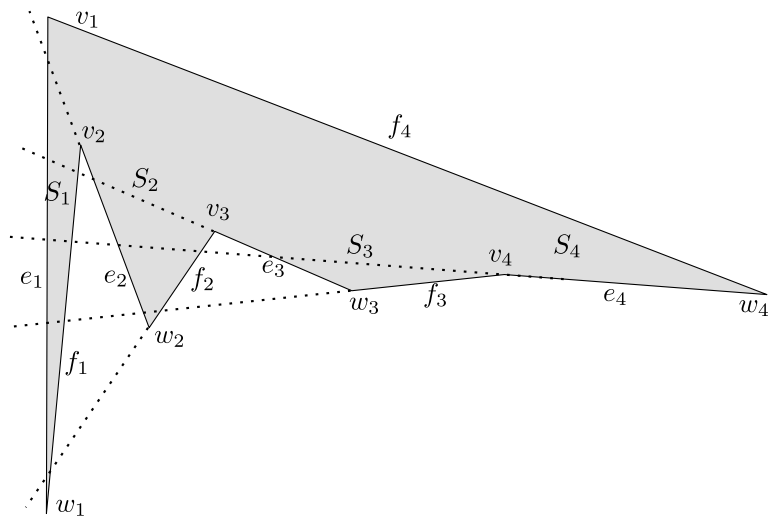


of the upper hyperbola in $v_{2}$ intersects the lower hyperbola, and proceed in this way. When reaching $w_{m}$, draw the last edge $f_{m}$ from $w_{m}$ to $v_{1}$ to close the polygon. Due to the convexity of the hyperbolas, $P_{m}$ has the claimed property.

No two edges of $P_{m}$ are collinear. Consider the line arrangement defined by the edges of $P_{m}$. No two lines intersect outside $P_{m}$, unless one of them is the line through $f_{m}$. This leads to the following observation.

Observation 14 In any guarding for $P_{m}$ every 2-guard that does not cover $f_{m}$ is a natural vertex guard.

In other words all the 2-guards lie on vertices of $P_{m}$ except for 2-guards that may lie on the line that bounds $P_{m}$ from above.

\subsection{Natural Guards}

Theorem 15 For any even natural number $n$ there exists a simple polygon with $n$ edges which requires at least $n-2$ natural guards.

We prove the theorem by counting the guards in an optimal solution. We say a guard belongs to a spike $S_{i}$ if it is a natural edge guard on $e_{i}$ or $f_{i}$ or if it is a natural vertex guard on $v_{i}$ or $w_{i}$. As only natural guards are allowed, every guard belongs to exactly one spike. The basic idea is that most spikes must have at least two guards which belong to them. Obviously every spike $S_{i}$ must have at least one guard which belongs to it, since the edge $e_{i}$ must be covered (Lemma 2).

Lemma 16 Consider a guarding $\mathcal{G}\left(P_{m}\right)$ using natural guards only, and let $i \in$ $\{1, \ldots, m-1\}$. If only one guard from $\mathcal{G}\left(P_{m}\right)$ belongs to $S_{i}$, then this guard must be on $v_{i}$ or on $e_{i}$. If there is no guard at $w_{i}$ nor a guard on $f_{i}$ in $\mathcal{G}\left(P_{m}\right)$, then both a guard at $v_{i+1}$ and a guard on $e_{i+1}$ are in $\mathcal{G}\left(P_{m}\right)$.

Proof Assume only one guard from $\mathcal{G}\left(P_{m}\right)$ belongs to $S_{i}$. It cannot be the natural edge guard on $f_{i}$, because this would leave $e_{i}$ uncovered (Lemma 2). If we had a guard at $w_{i}$ only, there would be no guard to distinguish a point near $v_{i}$ outside $P_{m}$ from a point near $v_{i+1}$ located inside $P_{m}$ and below the line through $f_{i}$ (see the two circles in Fig. 11). This proves the first part of the lemma. Now assume there are no guards at $w_{i}$ nor on $f_{i}$. Then to cover the edge $f_{i}$ there must be a vertex guard at $v_{i+1}$. Furthermore, the edge guard on $e_{i+1}$ is the only remaining natural guard to distinguish a point at the apex of $S_{i}$ near $w_{i}$ from a point located to the right of the apex of $S_{i+1}$ near $w_{i+1}$ and above the line through $e_{i+1}$ (depicted by two crosses).

This lemma immediately implies Theorem 15. Proceed through the spikes from left to right. As long as a spike has at least two guards which belong to it, we are fine. Whenever there appears a spike $S_{i}$ with only one guard, we know that there must be at least two guards in $S_{i+1}$ namely at $v_{i+1}$ and on $e_{i+1}$. Either there is a third guard that belongs to $S_{i+1}$, and thus both spikes together have at least four guards; or again we know already two guards in $S_{i+2}$. In this way, we can go on until we either find 
Fig. 11 A spike of $P_{m}$

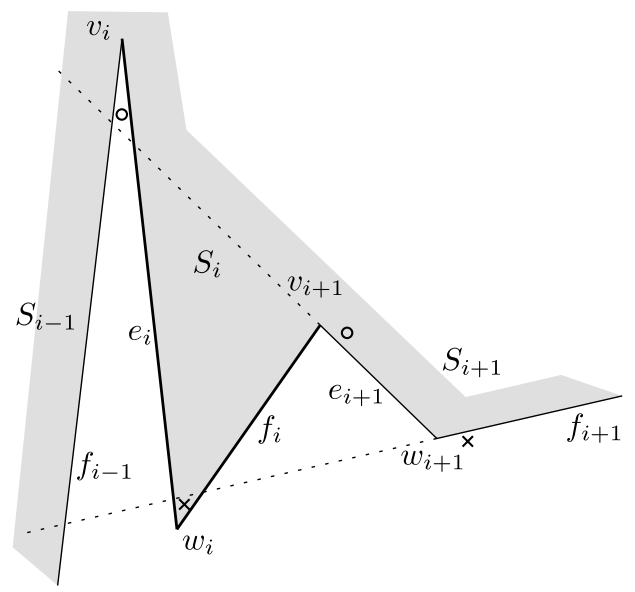

a spike which at least three guards belong to or we have gone through the whole polygon. So whenever there is a spike with only one guard either there is a spike with at least three guards that makes up for it, or every spike till the end has two guards. Hence there can be at most one spike guarded by one guard only that is not made up for later. For the last spike $S_{m}$ the lemma does not hold and we only know that it has at least one guard. So all in all there are at least $2(m-2)+1+1=n-2$ guards.

\subsection{General Guards}

Theorem 17 For any even natural number $n$ there exists a simple polygon with $n$ edges which requires at least $\lceil(3 n-4) / 5\rceil$ guards.

Before proving Theorem 17 let us note that we can find a guarding for $P_{m}$ using roughly $\frac{2}{3} n$ guards. Put a natural vertex guard $g_{1}$ on $v_{1}$ and $g_{2}$ on $v_{2}$, then put a nonnatural vertex guard $h_{2}$ on $w_{2}$ that guards $f_{2}$ with its right ray and whose left ray goes down vertically. Continue with a natural vertex guard $h_{3}$ on $w_{3}$ and an non-natural vertex guard $g_{4}$ on $v_{4}$ that guards $e_{4}$ with its right ray and with its left ray going up vertically. Then again put a natural vertex guard $g_{5}$ on $v_{5}$, a similar non-natural vertex guard $h_{5}$ on $w_{5}$ as before, a natural guard $h_{6}$ on $w_{6}$, a non-natural guard $g_{7}$ on $v_{7}$, a natural vertex guard $g_{8}$ on $v_{8}$, and so on. Then, $P_{m}$ can be described as $g_{1} \cap g_{2} \cap\left(h_{2} \cup h_{3} \cup\left(g_{4} \cap g_{5} \cap\left(h_{5} \cup h_{6} \cup(\ldots)\right)\right)\right)$.

Proof of Theorem 17 Consider a polygon $P_{m}$ as defined above, and let $\mathcal{G}\left(P_{m}\right)$ be a guarding for $P_{m}$. Define $a$ to be the number of 2-guards in $\mathcal{G}\left(P_{m}\right)$, and let $b$ be the number of other guards. All the $n$ edges of $P$ have to be covered somehow. An edge can be covered completely by a 2-guard, a 1-guard, or a 1'-guard. If no guard covers it completely, then the edge must be covered by at least two guards partly (Lemma 2). Moreover, at least one of these guards, namely the one covering the section towards the right end of the edge, is a $0^{\prime}$-guard, because the orientation cannot be correct to cover a second edge. So if an edge $e$ is not covered by a 2-guard, then there is at least one guard that does not cover any edge other than $e$. Therefore $2 a+b \geq n$. 


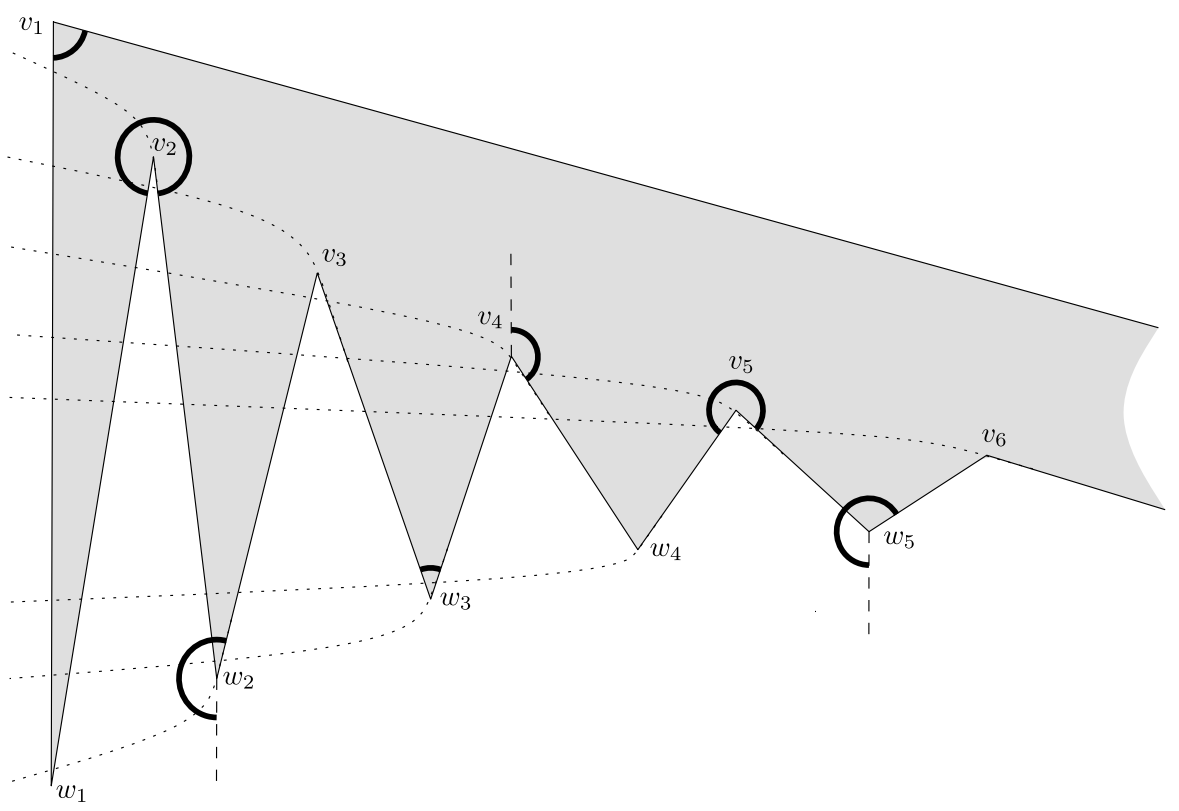

Fig. 12 A guarding for $P_{m}$ using roughly $\frac{2}{3} n$ guards, the dotted lines should be seen as straight lines

For any $i \in\{1, \ldots, m-2\}$ let $h_{i}$ be the directed line segment on $e_{i+2}^{-}$from the intersection of $e_{i+1}$ and $e_{i+2}^{-}$to $e_{i+2}$ (see Figs. 10 and 13). Similarly, let $h_{i}^{\prime}$ be the directed line segment from $w_{i+1}$ to the intersection of $f_{i+1}^{-}$and $f_{i}$.

As in Lemma 16 , consider pairs $\left(p_{1}, q_{1}\right), \ldots,\left(p_{m-2}, q_{m-2}\right)$ and $\left(p_{1}^{\prime}, q_{1}^{\prime}\right), \ldots$, $\left(p_{m-2}^{\prime}, q_{m-2}^{\prime}\right)$ of points infinitesimally close to the starting point or the endpoint of the corresponding line segment, located as follows: $p_{i}, p_{i}^{\prime} \in P_{m}$ for all $i, q_{i}, q_{i}^{\prime} \notin P_{m}$ for all $i, p_{i}$ is outside the natural vertex guard at $w_{i+1}$, whereas $q_{i}$ is inside the natural vertex guard at $w_{i+2}$, and similarly, $p_{i}^{\prime}$ is outside the natural vertex guard at $v_{i+2}$, whereas $q_{i}^{\prime}$ is inside the natural vertex guard at $v_{i+1}$. There are $n-4$ such pairs, and they need to be distinguished somehow (Observation 1). Any natural vertex guard can distinguish at most one pair, and the same is true for any (non-natural) 2-guard located along the line through $f_{m}$. Thus any 2-guard in $\mathcal{G}\left(P_{m}\right)$ distinguishes at most one of the pairs (Observation 14).

We claim that every guard $g$ in $\mathcal{G}(P)$ can distinguish at most three of these pairs. Denote the vertex of $g$ by $v_{g}$, and let $\ell_{g}$ and $r_{g}$ denote the left and right ray of $g$, respectively. Assume $g$ distinguishes $p_{i}$ from $q_{i}$. There are three cases: If $v_{g}$ is to the left of $h_{i}$, then-in order to distinguish $p_{i}$ from $q_{i}$ - the ray $r_{g}$ must intersect $h_{i}$. Symmetrically, if $v_{g}$ is to the right of $h_{i}$, then $\ell_{g}$ must intersect $h_{i}$. Finally, if $v_{g}$ is on the line through $h_{i}$ then it must be on the line segment $h_{i}$ itself. To distinguish $p_{i}$ from $q_{i}$, the endpoint of $h_{i}$ (i.e. $v_{i+2}$ ) must be inside $g$ (possibly on the boundary of $g$ ), hence $\ell_{g}$ must point to the left side of $h_{i}$ or in the same direction as $h_{i}$, and $r_{g}$ must point to the right side of $h_{i}$ or in the same direction. Since the claim is trivial for a degenerate guard with angle 0 , we can assume without loss of generality that at least one of the two rays is not collinear to $h_{i}$. 


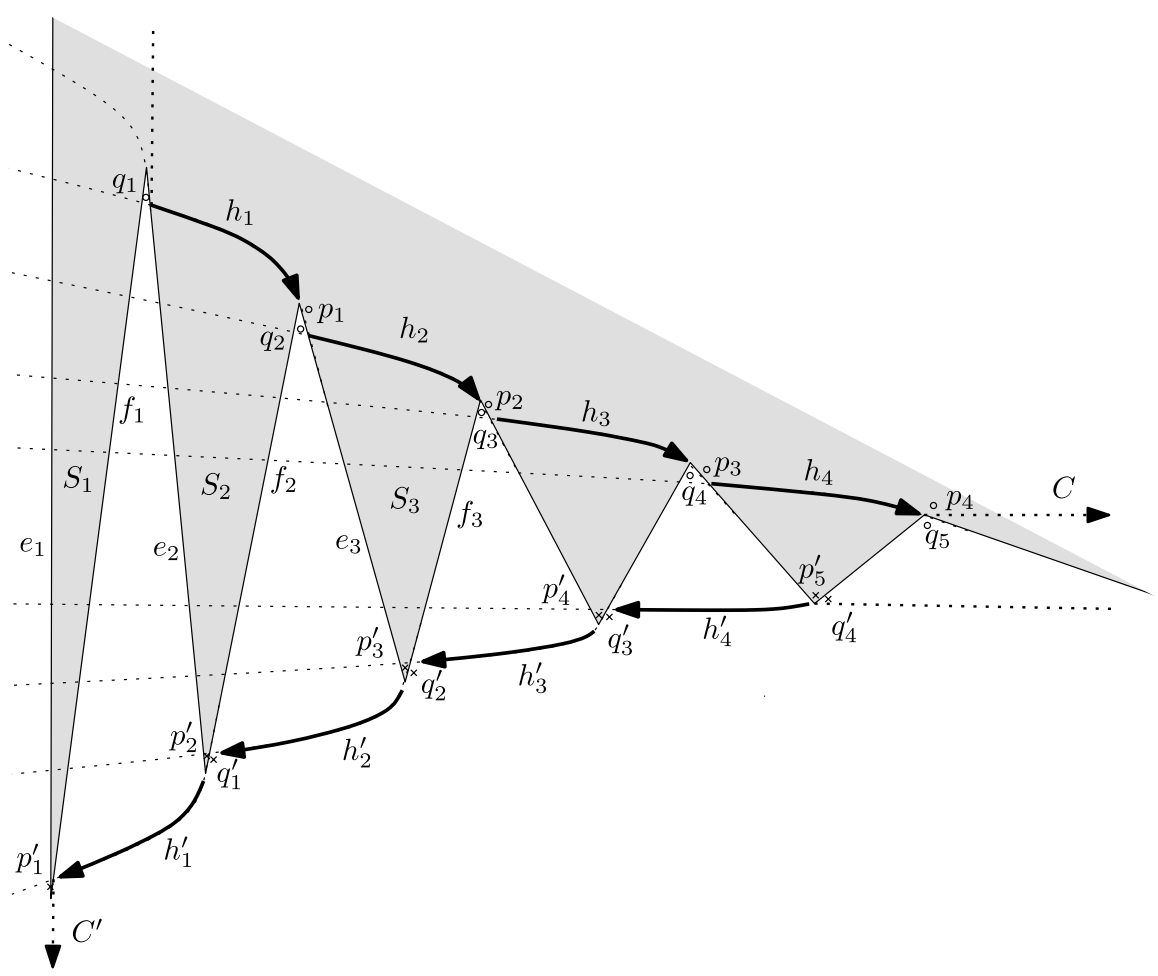

Fig. 13 The pairs $\left(p_{i}, q_{i}\right)$ and $\left(p_{i}^{\prime}, q_{i}^{\prime}\right)$ must be distinguished. See also Fig. 11. The directed line segments $h_{i}$ and $h_{i}^{\prime}$ form a convex curve $C$ and $C^{\prime}$, respectively

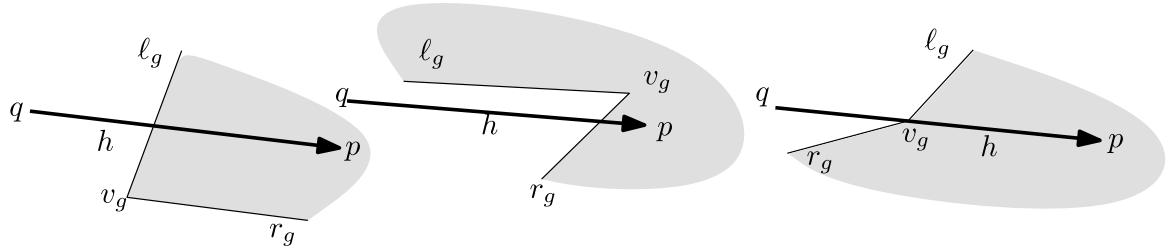

Fig. 14 Different ways $g$ can distinguish $p$ and $q$. In every case $\ell_{g}$ intersects $h$ leaving to the left side or $r_{g}$ intersects $h$ leaving to the right

Now assume $g$ distinguishes $p_{i}^{\prime}$ and $q_{i}^{\prime}$. Again there are three cases: If $v_{g}$ is to the right of $h_{i}^{\prime}$, then $\ell_{g}$ must intersect it, if it is to the left $r_{g}$ must intersect it. If $v_{g}$ lies on $h_{i}^{\prime}, \ell_{g}$ leaves to the left and $r_{g}$ to the right, or either or both rays lie on $h_{i}^{\prime}$.

In any case either $\ell_{g}$ intersects $h_{i}\left(h_{i}^{\prime}\right.$, respectively) coming from the right side of $h_{i}\left(h_{i}^{\prime}\right)$ and leaving to the left side, or $r_{g}$ intersects $h_{i}\left(h_{i}^{\prime}\right)$ coming from the left side and leaving to the right, or $\ell_{g}$ starts on $h_{i}\left(h_{i}^{\prime}\right)$ itself leaving to the left or $r_{g}$ starts on the line segment itself leaving to the right (see Fig. 14). If $r_{g}$ leaves an oriented line segment to the right side of the segment or if $\ell_{g}$ leaves an oriented line segment to the left side, we say the ray crosses the line segment with correct orientation. So 
Fig. 15 A guard $g$ with three and a guard $g^{\prime}$ with two correctly oriented crossings (marked with a circle)

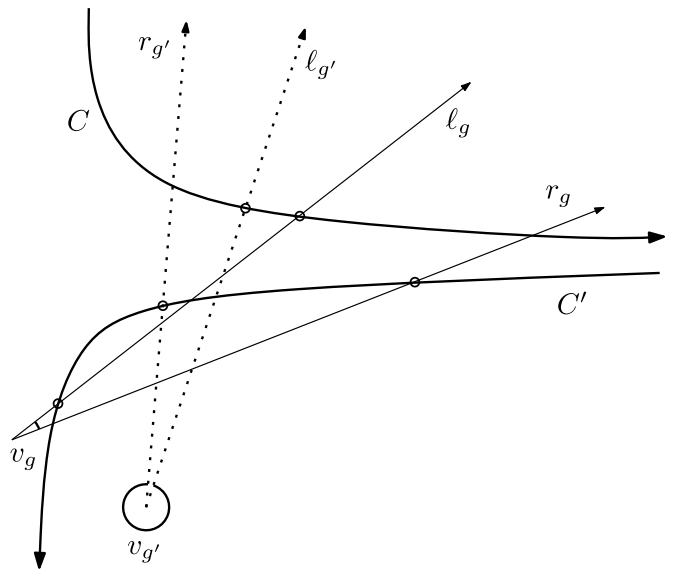

whenever a pair $\left(p_{i}, q_{i}\right)$ or $\left(p_{i}^{\prime}, q_{i}^{\prime}\right)$ is distinguished by $g$, then at least one of the rays $\ell_{g}$ or $r_{g}$ has a correctly oriented crossing with $h_{i}\left(h_{i}^{\prime}\right.$, respectively).

The line segments $h_{1}, \ldots, h_{m-2}$ lie on a oriented convex curve $C$, which we obtain by prolonging every line segment until reaching the starting point of the next one. Extend the first and last line segment to infinity vertically on the left and horizontally on the right. In the same way define a curve $C^{\prime}$ for $h_{1}^{\prime}, \ldots, h_{m-2}^{\prime}$ (see Fig. 13). Any ray can cross a convex curve at most twice. Because of the way $C$ and $C^{\prime}$ are situated with respect to each other (a line that crosses $C$ twice must have negative slope, a line that crosses $C^{\prime}$ twice must have positive slope) a ray can intersect $C \cup C^{\prime}$ at most three times. But we are only interested in crossings with correct orientation. If a ray crosses a curve twice, exactly one of the crossings has the correct orientation. If a ray crosses both $C$ and $C^{\prime}$ once, exactly one of the crossings has the correct orientation. Therefore any ray can have at most two correctly oriented crossings (see Fig. 15). If one of the rays $r_{g}$ or $\ell_{g}$ has two correctly oriented crossings, the other ray can have at most one. Thus both rays together can have at most three correctly oriented crossings and therefore distinguish at most three pairs. This leads to the second inequality $a+3 b \geq n-4$. Both inequalities together imply $a+b \geq \frac{3 n-4}{5}$.

\section{References}

1. Aigner, M., Ziegler, G.M.: Proofs from THE BOOK, 3rd edn. Springer, Berlin (2003)

2. Chvátal, V.: A combinatorial theorem in plane geometry. J. Comb. Theory Ser. B 18, 39-41 (1975)

3. Damian, M., Flatland, R., O'Rourke, J., Ramaswami, S.: A new lower bound on guard placement for wireless localization. In: 17th Annual Fall Workshop on Computational Geometry. http://arxiv.org/pdf/0709.3554v1 (2007)

4. Dobkin, D.P., Guibas, L., Hershberger, J., Snoeyink, J.: An efficient algorithm for finding the CSG representation of a simple polygon. Algorithmica 10, 1-23 (1993)

5. Eppstein, D., Goodrich, M.T., Sitchinava, N.: Guard placement for efficient point-in-polygon proofs. In: Proc. of the 23rd Annu. Sympos. Comput. Geom., pp. 27-36 (2007)

6. Fisk, S.: A short proof of Chvátal's watchman theorem. J. Comb. Theory Ser. B 24, 374 (1978)

7. Fragoudakis, C., Markou, E., Zachos, S.: Maximizing the guarded boundary of an art gallery is APXcomplete. Comput. Geom. Theory Appl. 38(3), 170-180 (2007) 
8. Guibas, L.J., Hershberger, J., Snoeyink, J.: Compact interval trees: a data structure for convex hulls. Int. J. Comput. Geom. Appl. 1(1), 1-22 (1991)

9. Kahn, J., Klawe, M.M., Kleitman, D.J.: Traditional galleries require fewer watchmen. SIAM J. Algebr. Discrete Methods 4, 194-206 (1983)

10. Karavelas, M.I.: Guarding curvilinear art galleries with edge or mobile guards. In: Proc. of the 13th ACM Solid and Physical Modeling Symposium, pp. 339-345 (2008)

11. Lee, D.T., Lin, A.K.: Computational complexity of art gallery problems. IEEE Trans. Inf. Theory 32(2), 276-282 (1986)

12. O'Rourke, J.: Galleries need fewer mobile guards: a variation on Chvátal's theorem. Geom. Dedicata 14, 273-283 (1983)

13. O'Rourke, J.: Visibility. In: Goodman, J.E., O'Rourke, J. (eds.) Handbook of Discrete and Computational Geometry, Chap. 28, pp. 643-663. CRC, Boca Raton (2004)

14. O'Rourke, J.: Computational geometry column 48. ACM SIGACT News 37(3), 55-57 (2006)

15. Shermer, T.C.: Recent results in art galleries. Proc. IEEE 80(9), 1384-1399 (1992)

16. Speckmann, B., Tóth, C.D.: Allocating vertex pi-guards in simple polygons via pseudo-triangulations. Discrete Comput. Geom. 33(2), 345-364 (2005)

17. Urrutia, J.: Art gallery and illumination problems. In: Sack, J.-R., Urrutia, J. (eds.) Handbook of Computational Geometry, pp. 973-1027. Elsevier Science/North-Holland, Amsterdam (2000) 